\title{
Cellulose Nanocrystal Interactions Probed by Thin Film Swelling to Predict Dispersibility
}

\author{
Michael S. Reid, Marco Villalobos and Emily D. Cranston
}

DOI: $10.1039 / C 6 N R 01737 \mathrm{~A}$

Reprinted (adapted) with permission from Reid M.S et al. Cellulose Nanocrystal Interactions Probed by Thin Film Swelling to Predict Dispersibility, Nanoscale, 2016,8, 12247-12257. Copyright (2016) Royal Society of Chemistry. 


\section{Journal Name}

\section{ARTICLE}

\section{Cellulose Nanocrystal Interactions Probed by Thin Film Swelling to Predict Dispersibility}

Received 00th January 20xx, Accepted 00th January 20xx

DOI: $10.1039 / x 0 x \times 00000 x$

www.rsc.org/

\author{
Michael S. Reid ${ }^{a}$, Marco Villalobos ${ }^{b}$ and Emily D. Cranston ${ }^{a,+}$
}

\section{Introduction}

Despite recent advances, reinforced polymeric nanocomposites (apart from elastomeric systems) are still in their infancy. Cost, poor particle dispersibility and limited load transfer between the phases have been major stumbling blocks within the field. The variety of matrices and particles available requires specific application design, however, ultimately both the mechanical and thermal properties of nanocomposites are governed by the balance between cohesive particle-particle and adhesive particle-polymer interactions. ${ }^{1}$ Indeed Akcora et al. were able to predict and control nanoparticle assembly in polystyrene matrices by altering the cohesive and adhesive interactions within the composite. ${ }^{2}$ Grafting increasingly dense polymer chains onto

\footnotetext{
a. Department of Chemical Engineering, McMaster University, Hamilton, Ontario, Canada, L8S 4 L8

${ }^{b .}$ Cabot Corporation, Billerica, MA, US, 01821

+Corresponding author: ecranst@mcmaster.ca

Details regarding CNC purification and characterization, XPS data of raw and extracted CNCs, AFM images of dry CNC films, fitting of SPR data and layer parameters, AFM images of water swollen CNC films, reference Hildebrand and Hansen solubility parameters for solvents tested, description of DLVO calculations. Are located in the ESI. See DOI: 10.1039/x0xx00000x
}

the nanoparticles reduced particle-particle interactions and assembly shifted from spherical aggregates to sheets to strings and finally individually dispersed nanoparticles. (Herein we define cohesion as the interaction between nanoparticles of the same material in contrast to the traditional definition of cohesion being within a material; this is also used in the powder and composites materials community. ${ }^{3}$ ) Advancing the field of reinforced nanocomposites requires methods to extract and predict these interactions in a variety of systems.

Cellulose nanocrystals (CNCs) are an abundant and sustainable rod-shaped nanomaterial that has recently been used in biomedical devices, ${ }^{4-6}$ as rheological modifiers, ${ }^{7,8}$ emulsion stabilizers ${ }^{9-12}$ and many other composite applications. ${ }^{13-17}$ Depending on the cellulose source (e.g., wood, cotton, bacteria, algae or tunicate) CNCs range from 100-1000 nm in length and 5-20 nm in cross-section and are commonly extracted through sulfuric acid hydrolysis. ${ }^{16,18,19}$ Although, applications span several fields, the potential for $\mathrm{CNCs}$ to act as reinforcing agents in polymer matrices is widely discussed. $^{15}$ Analytical models and experimental measurements report a longitudinal Young's modulus that ranges from $56-220 \mathrm{GPa}$ and a density of $1.5-1.6 \mathrm{~g} / \mathrm{cm}^{3} .{ }^{15}$

Currently, the major hurdle limiting CNC reinforced nanocomposites is the hydrophilic nature of the particles. High 
density of surface hydroxyl groups limits the compatibility with hydrophobic polymers leading to CNC aggregation and limited mechanical improvement. Furthermore, in the dried form (spray dried or freeze dried), CNCs can form strong agglomerates (sometimes attributed to hydrogen bonding) ${ }^{20}$ which are not easily overcome when CNCs are added to molten polymers or compounded using organic solvents. ${ }^{21-23} \mathrm{~A}$ significant amount of work has aimed to improve particlepolymer adhesion by esterification, silylation, urethanization, amidation, as well as grafting polymers to and from the CNC surface. ${ }^{24}$ Regardless of the CNC surface chemistry, the ability to extract and predict particle-particle and particle-polymer interactions in composites remains challenging due to nanoscale dimensions and poor colloidal stability in nonaqueous environments.

The objective of this work is to probe cohesive particleparticle interactions of sodium-form sulfuric acid-hydrolyzed CNCs, which are currently the type of CNCs most widely available commercially ${ }^{25}$ and as such, are the most industrially relevant for future CNC nanocomposite production. To this end, supported CNC thin films were swollen in various liquids to probe solvent uptake and particle-particle spacing. We find that the films swell in all solvents tested, the most in water and the least in acetone (with intermediate swelling observed for alcohols and acetonitrile), and in all cases films are held together by van der Waals forces. While qualitatively this is expected based on past fibre/cellulose studies, we believe that this is the first direct and quantitative report of probing CNCCNC interactions and comparing water and non-aqueous solvents.

Cellulose thin films have been commonly used to examine adhesion, adsorption and swelling of regenerated cellulose, cellulose nanofibrils and CNCs in water. ${ }^{26-33}$ With respect to pure CNC films, Kittle et $a .^{32}$ observed that swollen films contain $75 \%$ water by mass, nearly three times greater than earlier measurements by Aulin et al. ${ }^{30}$ Rationalizing the disparity between these measurements is challenging but is suggested to be due to the initial state of hydration in the film. ${ }^{32}$ In the most recent study, Niinivaara et al. monitored CNC film swelling in humid environments by quartz crystal microbalance with dissipation (QCM-D) and spectroscopic ellipsometry. ${ }^{33}$ They deduced that hydrated CNCs retain a 2 $\mathrm{nm}$ water layer around each CNC particle. While these works provide critical insight into CNC film swelling in aqueous environments, there remains poor agreement across studies and a lack of understanding of these interactions in nonaqueous liquid media.

Our work uses surface plasmon resonance spectroscopy (SPR) which is less common than ellipsometry and QCM-D, and is an optical technique that monitors the intensity of a laser beam reflected off a plasmonic surface (gold or silver); we show here that it offers quantitative insight into the interactions between nanoparticles, in a straightforward way. Unlike most other techniques, SPR is capable of tracking in situ the transition from the dry to the liquid swollen state. SPR is regularly used as a biosensor to measure adsorption or desorption (and binding kinetics) and although it has been used to probe various cellulose surfaces in aqueous environments $^{34-44}$ some SPR instrumentation is more suited to materials science investigations, such as the equipment used here, where film thickness and density are obtained from optical fitting of SPR reflectivity over a large angular range. This is in contrast to instruments that only work over a small angular range and, for example, cannot be used in media with different refractive indices $(n)$ from water. Despite the fact that full angle SPR can operate in a number of solvents, the operational range for our films is generally limited to solvents with refractive indices below $n=1.4$, excluding typical wettability test solvents, such as methylene iodide $(n=1.530)$.

In a recent study also based on SPR, Kontturi et al. monitored water content of highly porous cellulose nanofibril films, which when swollen contained over $50 \%$ water, agreeing with parallel QCM-D measurements. ${ }^{29}$ Importantly, the authors note that sample heterogeneity within the cellulose nanofibril film made SPR measurements and data fitting very challenging. Here we test smooth and uniform CNC films in a variety of suitable SPR solvents: water, acetone, methanol, acetonitrile, isopropanol, and ethanol, and although they do not represent common hydrophobic polymers in terms of their solubility parameters (i.e., polyethylene or polystyrene), they do mimic polymers aimed towards biodegradable composites and biomedical applications such as poly(vinyl alcohol), poly(lactic acid) and poly(vinyl pyrrolidone).

This paper presents SPR (with supporting results from atomic force microscopy, AFM) as a novel method to probe cohesive particle-particle interactions through film swelling and relate nanoparticle dispersibility to Hildebrand and Hansen solubility parameters. We believe this technique and our approach is applicable to other nanoparticle systems and can offer significant insight for nanoparticle dispersion and nanocomposite design. The study of CNC films specifically, differs from studying the properties of individualized CNCs in aqueous suspension ${ }^{18,45}$ or in polar-organic solvents ${ }^{46}$ as CNC interactions within the film are expected to represent aggregated dried CNCs which is the commercially available format. Overall, we demonstrate that CNC particle-particle interactions are strong and are dominated by hydrogen bonding and attractive van der Waals forces.

\section{Experimental}

\section{Materials}

Whatman cotton ashless filter aid was purchased from GE Healthcare Canada. Sulfuric acid, methanol, acetone, acetonitrile and isopropanol were all obtained from Caledon Laboratory Chemicals (Georgetown, ON, Canada). Anhydrous ethanol was purchased from Commercial Alcohols (Brampton, ON, Canada). Hydrogen peroxide was purchased from Sigma Aldrich (Oakville, ON, Canada). All chemicals were used as received. Water used was purified Type I water with a resistivity of $18.2 \mathrm{M} \Omega \cdot \mathrm{cm}$ (Barnstead NANOpure Dlamond system, ThermoScientific, Asheville, NC). 


\section{Sulfuric Acid Hydrolysis of CNCs}

CNCs were prepared by sulfuric acid hydrolysis, as previously described. ${ }^{47}$ Briefly, $40 \mathrm{~g}$ of cotton Whatman ashless filter aid was digested in $700 \mathrm{~mL}$ of $64 \mathrm{wt} . \%$ sulfuric acid at $45^{\circ} \mathrm{C}$ for 45 min under continuous mechanical stirring. Immediately following hydrolysis, the mixture was diluted 10 -fold with $4^{\circ} \mathrm{C}$ water to quench the reaction. The suspension was centrifuged at $6000 \mathrm{rpm}$ for $10 \mathrm{~min}$ four times to remove excess acid. The precipitate was then diluted and dialysed against water until the $\mathrm{pH}$ stabilized ( 2 weeks). The suspension was sonicated (Sonifier 450, Branson Ultrasonics, Danbury, CT) continuously in an ice bath for 15 min three times at $60 \%$ output. The CNC suspensions were neutralized (converted to the sodium salt form) by the addition of an appropriate amount of $1 \mathrm{mM}$ $\mathrm{NaOH}$ and then lyophilized for storage. Although work has shown that CNCs can adsorb organic material during production and from the environment ${ }^{48}$ we observed an insignificant reduction in the aliphatic carbon composition for spin coated CNC films before and after soxhlet extraction (ESI Table S1). We believe that environmental contamination during sample preparation cannot be avoided and as a result, CNCs used in this study were not purified further following dialysis. The CNC dimensions were $4-15 \mathrm{~nm}$ by $50-306 \mathrm{~nm}$, measured by AFM of 100 particles with an average cross section and length of $8 \mathrm{~nm}$ and $122 \mathrm{~nm}$ respectively. The surface sulfate half ester content was $0.7 \%$ sulfur by mass corresponding to approximately 0.41 charge groups per $\mathrm{nm}^{2}$ of CNC surface area as determined by conductometric titration with $\mathrm{NaOH} .{ }^{49}$

\section{Film Preparation}

CNC films were prepared on polished Si wafers (MEMC Electronic Materials Sdn Bhd, Petaling Jaya, Malaysia) or $\mathrm{SiO}_{2}$ coated SPR sensors (BioNavis, Ylöjärvi, Finland) by spin coating under $\mathrm{N}_{2}$ gas (G3P Spincoat, Specialty Coating Systems Inc. Indianapolis, USA) at $4000 \mathrm{rpm}$ for $30 \mathrm{~s}$ with a $7 \mathrm{~s}$ ramp. Prior to deposition, all surfaces were cleaned in a piranha solution (3:1 concentrated sulfuric acid to hydrogen peroxide) for 30 min, followed by continuous rinsing with purified water and drying by a stream of $\mathrm{N}_{2}$ gas. Suspensions were prepared by dispersing freeze dried CNCs in water and sonicating at $60 \%$ amplitude for $30 \mathrm{~s}$. Film thicknesses were controlled by varying the CNC suspension concentration between 1 and 3 wt.\%. Following spin coating, films were heat treated at $80^{\circ} \mathrm{C}$ for 8 hours (overnight) to remove water from the film. Films were then gently rinsed with purified water and heat treated again for 8 hours. We believe that this procedure removes loosely bound CNCs from the surface and improves the overall stability of the film by removing excess water, which increases particle-particle interaction by replacing water-CNC hydrogen bonds with CNC-CNC hydrogen bonds as discussed by Beck et al. ${ }^{21}$ Additionally we propose this procedure helps to eliminate radial ordering of CNCs which may occur during spin coating (as evidenced by AFM imaging).

\section{Atomic Force Microscopy}

AFM images were collected in alternating current (AC) mode using an Asylum MFP-3D instrument (Asylum Research an Oxford Instrument Company, Santa Barbara, CA). Images of dry films were collected in air under ambient conditions. Rectangular FMR cantilevers (NanoWorld) with normal spring constants of 1.2-5.5 N/m and resonant frequencies of 60-90 $\mathrm{kHz}$ were used for all morphological and scratch height measurements. Root-mean-squared (rms) roughness was calculated by averaging $251 \mu \mathrm{m} \times 1 \mu \mathrm{m}$ areas over a $5 \mu \mathrm{m} \times 5$ $\mu \mathrm{m}$ image. The thickness of dry CNC films was determined by in situ AFM scratch height analysis. Scratching was performed using the AFM tip in contact mode (with a set point of ca. $1 \mathrm{~V}$ or $200 \mathrm{nN}$ ) to gently remove CNCs from the surface over a 1 $\mu \mathrm{m} \times 1 \mu \mathrm{m}$ area. The force used was sufficient to displace CNCs but leave the underlying $\mathrm{SiO}_{2}$ substrate undamaged. The scratched area was reimaged $(5 \mu \mathrm{m} \times 5 \mu \mathrm{m})$ in AC mode, using the same tip used to scratch, to obtain the cross sectional profile. Images were processed in Igor Pro 6.0 running Asylum Research AFM software (version 13.17) using a second order flatten routine, excluding the scratched region. Thicknesses were measured by taking the average height difference from the top of the CNC film and the underlying substrate.

Swollen films were measured in purified water using an open fluid cell. Prior to swelling, dry films were scratched as described above and the cross section was measured. Water was then introduced and the system was allowed to equilibrate $(\sim 30 \mathrm{~min})$. The previously scratched areas were then reimaged $(5 \mu \mathrm{m} \times 5 \mu \mathrm{m})$ and the height of the film was determined. Film thickness values reported are from three separate films and images were processed in the same fashion as dry films and the data error bars represent the standard deviation of the initial film thickness.

\section{Surface Plasmon Resonance}

Full angle $\left(40^{\circ}-77^{\circ}\right)$ SPR curves were collected using a SPR $\mathrm{Navi}^{\mathrm{TM}} 200$ (BioNavis, Ylöjärvi, Finland). Laser wavelengths of $785 \mathrm{~nm}$ and $670 \mathrm{~nm}$ with a spot size of $500 \mu \mathrm{m} \times 500 \mu \mathrm{m}$ were simultaneously used to continuously monitor dry and swollen $\mathrm{CNC}$ films. Films were swollen under constant solvent flow rates of $100 \mu \mathrm{L} / \mathrm{min}$ at $25^{\circ} \mathrm{C}$ and allowed to equilibrate for 30 min. Within the flow cell an O-ring confines the film in the $x y$ (lateral) plane and thus films are assumed to only swell in the z-direction (vertical to the sensor surface). Experimental SPR curves were fit by the Fresnel equations using recursion formalism and assuming parallel slab geometry with the free software Winspall 3.01 (Max-Planck Institute for Polymer Research, Mainz, Germany). We note that the SPR signal is averaged over the laser spot and that film roughness and thickness will vary over the sensor surface (surface roughness by AFM is $5.7 \mathrm{~nm}$ over $1 \mathrm{um}^{2}$ ). While the precision in the SPR curve fitting and the relative changes in thickness are indeed at the Angstrom level, the overall accuracy in thickness is likely in the nanometer range. 
The refractive index $(n)$ and the thickness $(d)$ of dry CNC films were independently determined following the multiwavelength SPR approach as described by Granqvist et al. ${ }^{50}$ It is well known that when optically fitting layer parameters the $n$ and $d$ correlate to each other and form a $n-d$ continuum of possible solutions, but by employing both $670 \mathrm{~nm}$ and $785 \mathrm{~nm}$ wavelengths simultaneously, two sets of $n-d$ continua are established from which a unique solution for $n$ and $d$ can be obtained. Bare $\mathrm{SiO}_{2}$ sensors were initially modeled to determine background layer parameters and $n-d$ continua were calculated by modeling CNC film thickness for refractive indices between 1.30 and 1.55 with 0.005 intervals. Plotting the two continua on the same axis determined the intersection point and solutions for $n$ and $d$. Assuming CNC films behave as Cauchy materials, when used, the chromatic dispersion relation $(d n / d \lambda)$ of pure cellulose was taken to be $0.0271 \mu \mathrm{m}^{-1}$ and used to shift the $670 \mathrm{~nm}$ continuum. ${ }^{29,51}$ Notably $d n / d \lambda$ for air was considered negligible. Both shifted and non-shifted continua displayed intersection points and the difference between the two is taken as the uncertainty in the fitting and is discussed below. Within this study, the multi-wavelength approach was only applied to dry CNC films. The refractive index of CNCs is reported to range from $1.51^{32}$ to $1.62^{47}$ and is taken as $n_{C N C}=1.55$ within this work.

\section{Optical Theory and Thin Film Analysis}

In situ film swelling has been extensively studied by optical techniques, in particular via ellipsometry. ${ }^{33,52}$ However, extracting parameters such as thickness, refractive index and volume fraction of swollen films remains challenging and can contain a significant degree of error. For example, failure to account for the change in free volume within polymer films can lead to thickness errors in excess of $100 \%$ when using an effective medium approximation. ${ }^{53}$ Additionally, depending on the penetrant, swelling can occur following either constant or volume additive regimes convoluting which optical models are appropriate. ${ }^{54}$ Considering the volume additive regime: as the film swells the volume fraction of the swollen material decreases, reducing the effective refractive index of the system. As a result, an inverse relationship develops between film thickness and refractive index. This was successfully employed by Erdoğan et al. who measured polymer film swelling via solvent vapour sorption using fixed angle SPR. ${ }^{55-57}$ In their study, the ratios of the volume $(V)$ and optical intensity $(I)$ from the initial to the equilibrium swollen state were inversely proportional according to Equation 1:

$$
\frac{V_{E q}}{V_{t}}=\frac{I_{t}}{I_{E q}}
$$

where subscripts, $t$ and $E q$ refer to the film at time, $t$, and at equilibrium, respectively. It is important to note that because the refractive index of solvent vapour does not differ significantly from air the bulk refractive index of the system remains constant. As a result, angular shifts in the SPR peak are relatively small $\left(<1^{\circ}\right)$ and fixed angle SPR is applicable. ${ }^{55}$ When liquid swelling agents are used the bulk refractive index of the system radically changes, significantly shifting the SPR peak position $\left(>25^{\circ}\right)$. Full angle SPR measurements are capable of accounting for large angular changes

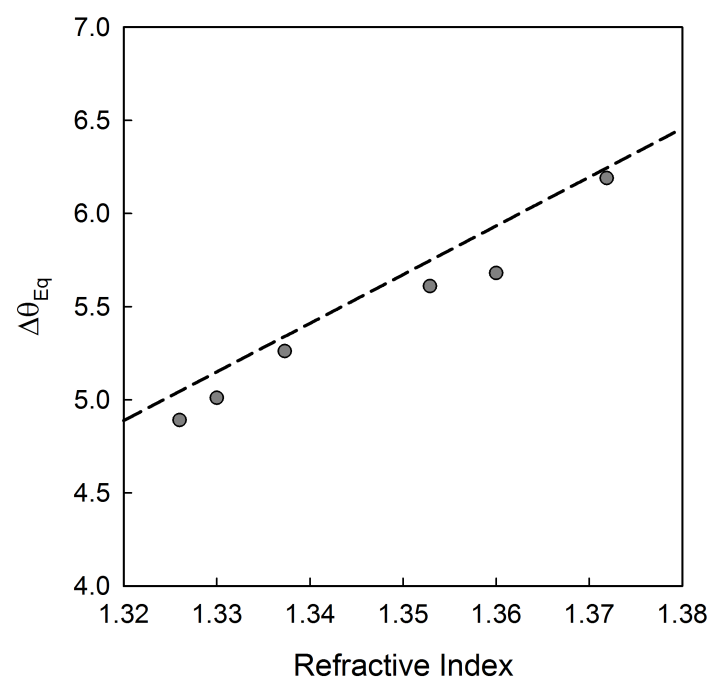

Figure 1: Experimental (circles) and modeled (dashed line) shift in SPR angle with solvent refractive index.

and thus swelling information can be extracted. In the present study, the significant increase in angular position often shifted the $670 \mathrm{~nm}$ SPR peak outside the measurable range, eliminating the possibility for a multi-wavelength approach. As a result, CNC films were characterized by the difference in angular position of the 785 $\mathrm{nm}$ SPR peak and total internal reflectance peak (TIR) in an adapted form of Equation 1:

$$
\frac{V_{E q}}{V_{o}}=\frac{\Delta \theta_{o}}{\Delta \theta_{E q}}
$$

where $\Delta \theta_{\mathrm{o}}$ and $\Delta \theta_{\mathrm{Eq}}$ are the difference between the SPR peak and TIR angles for the initial dry and swollen films, respectively. Critically, $\Delta \theta_{\mathrm{Eq}}$ must be corrected to account for the solvent refractive index to isolate angular shifts due to CNC film swelling alone; contrary to the common description of SPR it is not just the TIR that shifts when the media refractive index changes but the SPR peak as well. Figure 1 presents modeled (dashed line) and experimentally measured $\Delta \theta_{\mathrm{Eq}}$ values for a bare $\mathrm{SiO}_{2}$ coated SPR sensor in various solvents. Within the range explored, there is a linear increase of $\Delta \theta_{\mathrm{Eq}}$ with solvent refractive index. This phenomenon is rarely discussed in literature and to our knowledge Figure 1 is the first explicit presentation of this effect. ${ }^{58}$ Failure to subtract solvent effects from $\Delta \theta_{\mathrm{Eq}}$ would artificially increase the perceived swelling in thin films.

\section{Results}

\section{CNC Films in Air}

The thickness and morphology of CNC thin films in air were measured by AFM and SPR (Figure 2) to compare the results of physical versus optical methods. AFM images showed that complete, uniform films were produced with an average roughness of $5.7 \pm 0.4 \mathrm{~nm}$ (Figure 2a). Additionally, Figure 2a shows that the CNC films are isotropic in the xy plane, indicating that the alignment often observed in spin coated CNC films has been eliminated due to the "heat treat-rinse-heat treat" protocol. ${ }^{47,59,60}$ Figure $2 \mathrm{~b}$ and Figure $2 c$ present the scratched AFM image and the average cross sectional analysis of the film, respectively. The scratched image 

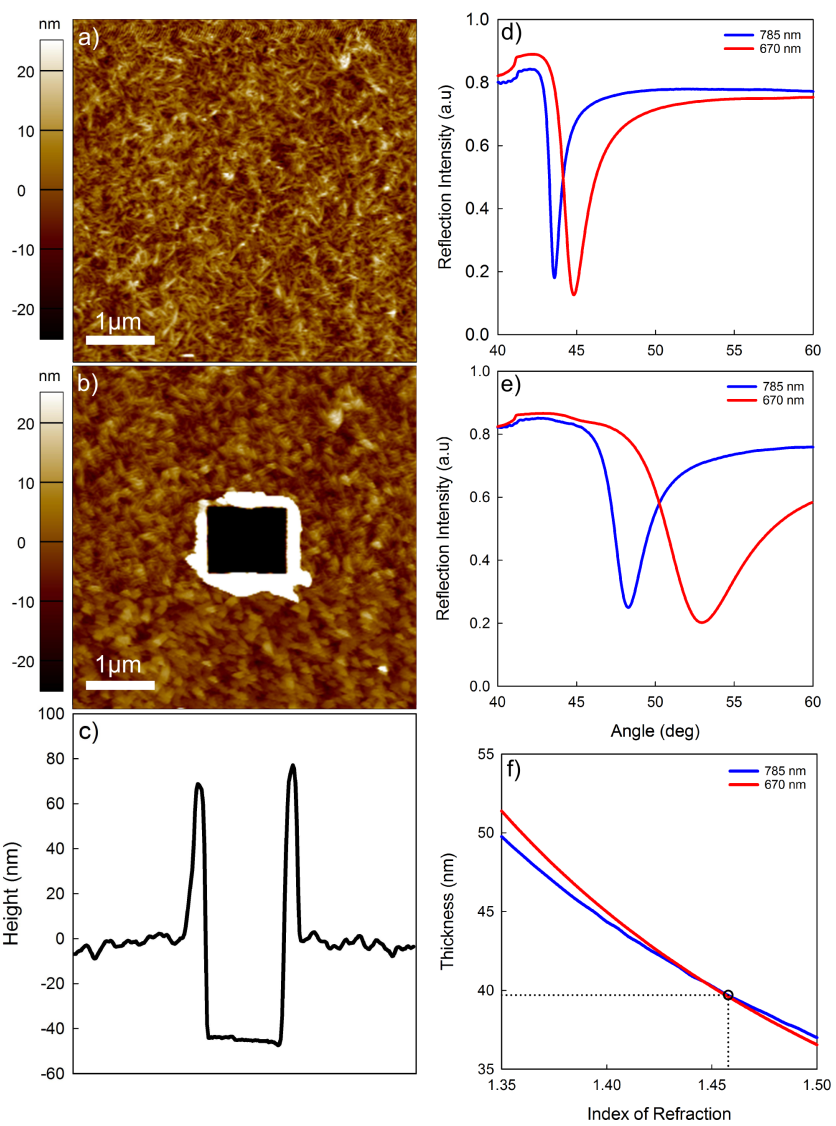

Figure 2: AFM height image of (a) a dry CNC film after the "heat treat-rinse-heat treat" procedure, (b) scratched CNC film, and (c) cross section height analysis of scratched image used to determine film thickness. SPR spectrum of (d) bare $\mathrm{SiO}_{2}$ substrate and (e) dry CNC film. Wavelength crossover (f) displays an index of refraction of 1.4578 and a thickness of 39.7 $\mathrm{nm}$.

shows that CNCs have been removed from the substrate and that the displaced CNCs have built-up in the region immediately surrounding the scratched area. Amplitude and phase images indicate that no CNCs remain in the scratched region (ESI Figure S1) and an average film thickness of $40 \pm 5 \mathrm{~nm}$, corresponding to 4-5 CNC layers, was calculated as the height difference between the bare substrate and the unaffected CNC film region.

Similarly, CNC films were characterized by multi-wavelength SPR, ${ }^{50}$ (Figures $2 \mathrm{~d}$ and $2 \mathrm{e}$ ) and data were fit following the Fresnel equations by adjusting the thickness $(d)$, refractive index $(n)$ and extinction coefficient $(k)$ of each layer using Winspall software (see ESI Figure S2 and Figure S3 for layer model parameters). Figure $2 d$ displays characteristic features of a typical full angle SPR curve for a clean sensor, which includes an increase in reflected light intensity at $41^{\circ}$ due to the TIR and a significant reduction in intensity at the SPR angle. Figure 2e displays the same features however, the SPR peak has shifted to higher angles due to the presence of CNCS at the sensor surface. ${ }^{29}$ In addition, the breadth of the SPR peak increases following CNC deposition indicating that the roughness at the air interfaces has increased, correlating with AFM roughness measurements. ${ }^{34}$

Figure $2 \mathrm{f}$ presents the $n$ - $d$ continuum from which $d$ and $n$ of the film were determined. Assuming the chromatic dispersion $(d n / d \lambda)$ is negligible, an intersection point is observed at $d=39.7 \mathrm{~nm}$ and $n=$
1.458 correlating well with AFM thickness measurements. Applying

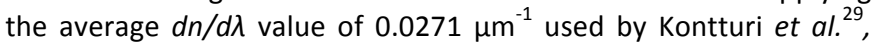
the layer parameters shifted to $d=39.1 \mathrm{~nm}$ and $n=1.466$. It is important to note that the $d n / d \lambda$ value was determined for pure cellulose, the structure of which was not specified and potentially contains both amorphous and crystalline material, ${ }^{51}$ thus it is unclear if this value can be directly applied to CNCs which are composed of crystalline cellulose 1. ${ }^{59}$ Additionally, the porous nature of the films suggests the $d n / d \lambda$ cannot be modeled simply as a single phase but as a weighted average between air and cellulose. As such, the difference between the values with and without the $d n / d \lambda$ factor is taken to be the uncertainty in the film thickness and effective refractive index, yielding parameters of $d=39.7 \pm 0.6 \mathrm{~nm}$ and $n=1.458 \pm 0.008$.

Because the CNC films are not free standing and are very thin, calculating the density is challenging. However, the effective refractive index of 1.458 indicates that the films contain a high density of CNCS $\left(n_{C N C}=1.55\right)$ and Braun and Pilon showed volume averaging theory (VAT) can be used to calculate the porosity $(\phi)$ for non-adsorbing nanoporous thin films according to Equation $3:{ }^{61}$

$$
n_{f i l m}=\sqrt{(1-\phi) n_{C}^{2}+\phi n_{D}^{2}}
$$

where $n_{\text {film }}, n_{C}$, and $n_{D}$ are the refractive indices of the film, continuous phase and discontinuous phase, respectively. In the dry state, CNCs are in physical contact and are assumed to be the continuous phase thus $n_{C}=n_{C N C}$. Rearranging Equation 3 , the composition of the spin coated CNC film was calculated to be $20 \pm$ $2 \%$ air and $80 \pm 2 \%$ CNC by volume. This is in excellent agreement with similarly prepared CNC films by Niinivaara et al. ${ }^{33}$

\section{CNC Film Swelling}

CNC films were swollen in situ in the SPR by introducing various solvents under constant flow; the films remained stable throughout all measurements and no material was lost as evidenced by rinsing and drying at the end of each experiment to ensure the same SPR profile in air (i.e., Figure 2e) was obtained. While adsorbed atmospheric water may be present in the CNC films, all measurements were performed under the same conditions and all solvents tested are miscible with water thus we expect the surface of the particles to participate in a solvent exchange over the $30 \mathrm{~min}$ swelling period. Figure 3 presents the volume fraction of air or solvent in dry and swollen films, respectively, as measured by SPR. Intuitively, water exhibits the highest volume fraction of solvent within swollen films due to the hydrophilic nature of CNCs. Less space within the CNC films is created by swelling in non-aqueous solvents with the volume fraction increasing in the order of acetone, methanol, acetonitrile, isopropanol, and ethanol. A Student's t-test indicates that swelling in methanol, acetonitrile, isopropanol and ethanol is statistically equivalent. All swelling values differ from acetone $(p>0.2)$ and water $(p>0.05)$. The extent of swelling and the trends with respect to common physical parameters/constants are discussed further below.

To support SPR swelling measurements, AFM scratch height analysis was used to physically measure CNC film thickness, first in air (similar to Figure 2) and then in water using an open fluid cell, allowing the films to swell until equilibrium (ESI Figures S4 and S5). SPR swollen film thicknesses were calculated by assuming the volume increases only in the vertical direction. The SPR data error bars are calculated from the uncertainty in the volume percentage determined from the multi-wavelength SPR approach and leads to 


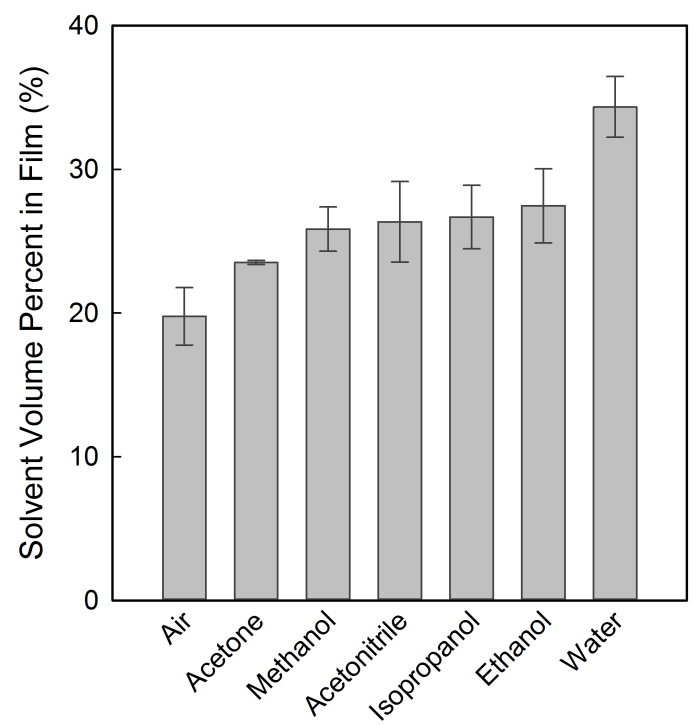

Figure 3: Percent volume of solvent in swollen CNC films.

larger uncertainty in thicker films. Figure 4 presents the change in film thickness (from dry to liquid environments) for CNC films with different starting thicknesses as measured by SPR and AFM. Plotting the percentage change in film thickness indicates that film swelling is statistically independent of starting film thickness (ESI S5). Over a range of dry film thicknesses $(25-70 \mathrm{~nm})$ the volume increased by an average of $13.5 \pm 0.4 \%$ measured by AFM and $14 \pm 2 \%$ by SPR. This highlights that the two methods correspond closely, and that swelling needs to be normalized by dry film thickness (which is already done for data presented in Figure 3). We also note that as indicated by Fält et al. ${ }^{62}$ it is difficult to avoid tip penetration during wet AFM imaging, but the agreement supports the interpretation of both techniques and that the volume fraction of CNC films can be measured by SPR using an inverse relationship with $\Delta \theta$. These results imply that this data processing approach can be extended to other solvents.

As a final test, water-swollen CNC films were monitored by SPR under increasing flow rates. The SPR peak angle exhibited no change for flow rates of $100-500 \mu \mathrm{L} / \mathrm{min}$, equivalent to shear stress of $0.3-1.4$ dyne $/ \mathrm{cm}^{2}$ (data not shown). ${ }^{63}$ This indicates that despite the large swelling of CNC films in water, it is not possible to partially or fully remove the CNCs from the substrate using the magnitude of shear available within the SPR instrument. Additionally, this measurement was conducted over a period of 2 hours from which negligible change in film thickness was observed after $30 \mathrm{~min}$.

\section{Discussion}

Particle dispersibility ultimately depends on the balance between adhesive particle-solvent or particle-polymer and cohesive particleparticle interactions. Here we present a method to measure CNC cohesion by looking at nanoparticle separation in a variety of solvents in which the particles are not colloidally stable. The results presented above indicate that CNC-CNC cohesive interactions remain strong in all of the solvents tested, as none of the films delaminated upon exposure to liquid. However, the interactions between particles can be partially screened which leads to film swelling. We believe that the CNC films studied here are a reasonable model for $\mathrm{CNC}$ agglomerates which may be present

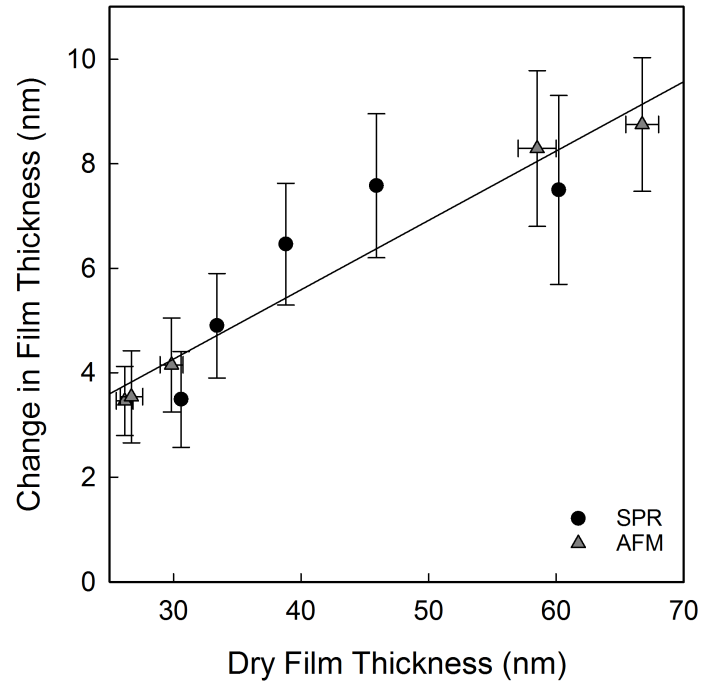

Figure 4: Change in CNC film thickness in water measured by SPR and AFM. Solid line is included as a guide for the eye.

during nanocomposite compounding and measurement of the cohesive interactions will prove useful for future formulation development. We emphasize that CNCs are insoluble in the solvents used here, however the interpretation of solubility parameters as "dispersibility parameters" for insoluble nanoparticles combined with SPR swelling will allow us to rationalize and predict CNC-CNC cohesive interactions in aqueous and non-aqueous environments.

\section{Film Swelling and Solubility Parameters}

Due to the hydrophilic nature of cellulose, swelling in water is of great interest ${ }^{26}$ and as expected, water was found to swell CNC films the most (followed by alcohols, acetonitrile, and acetone). Although cellulose fibers are known to swell in water, experimental $^{64}$ and theoretical ${ }^{65}$ work has demonstrated that solvent does not penetrate the cellulose I crystal structure of CNCs. As such, we assume that CNC films swell by increasing inter-particle spacing and not by increasing the size of individual particles. We predict that CNC film swelling follows two distinct steps: firstly, solvent rapidly penetrates the film through the porous structure (capillary action), filling the voids. Subsequent swelling occurs as solvent enthalpically wets CNC surfaces and continues to fill, and create new space between CNC particles. The new space created increases the thickness and total volume of the film by reducing cohesive particle-particle interactions. For solvents with hydrogen bonding capabilities, this wetting replaces the majority of particleparticle hydrogen bonds with particle-solvent hydrogen bonds. ${ }^{66}$

Water-swollen CNC films were determined to have a total thickness increase of $14 \pm 2 \%$ compared to the dry state. In contrast, films in non-aqueous solvents all showed a minimal change in volume; acetone increased the film volume by only $4 \%$ and methanol, acetonitrile, isopropanol and ethanol increased the volume by $6-8 \%$. There is no apparent correlation with the solvent dielectric constants which are 20.7, 32.7, 37.5, 17.9, and 24.5 for acetone, methanol, acetonitrile, isopropanol, and ethanol, respectively. This is similarly reflected in the lack of trend with cellulose-solvent Hamaker constants (approximated using Lifshitz theory, ESI Figure S6a), suggesting that swelling is not dependent on solvent shielding of van der Waals forces. Moreover, no trend 
corresponding to solvent molecular size was observed, eliminating any explanation based on sterics/solvation forces, and suggesting that the chemical nature of the solvent is responsible for interrupting particle-particle interactions and overall film swelling.

Swollen films contain $25 \pm 2$ wt.\% water, in good agreement with QCM-D measurements of similar swollen CNC films studied by Aulin et $a l^{30}$ and Niinivaara et $a l^{33}$ (assuming a CNCs density of 1.55 $\left.\mathrm{g} / \mathrm{cm}^{3}\right) .{ }^{15}$ In contrast, Kittle et al. determined water content of CNC films to be nearly triple this value at 74 wt.\% via QCM-D. ${ }^{32}$ Similar results were observed in low-density cellulose nanofibril films studied by Kontturi et al. ${ }^{29}$ Currently it is unclear as to why this discrepancy in the literature exists but highlights that film structure and the initial state of hydration can significantly alter swelling behaviour of nanocellulose films. The agreement of this work and similarly prepared CNC films by Aulin et al. and Niinivaara et al. supports our interpretation of SPR data and additionally the validity of SPR as useful tool in film swelling studies of nanoparticle systems.

To understand the dominant chemical properties that influence film swelling we turned to solubility parameters; the Hildebrand solubility parameter has long been used to predict solvation and polymer-solvent interactions based on cohesive energy density, ${ }^{67}$ and it may offer insight into insoluble particle-solvent interactions as well. Figure 5 indicates that there is a correlation $\left(R^{2}=0.9068\right)$ between solvent volume fraction taken up by the film and the Hildebrand solubility parameter; essentially, the larger the solubility parameter, the greater the particle-particle spacing (i.e., more swelling). This correlation is stronger than that between film swelling and solvent surface tension (see ESI Figure S6b) as well as Hamaker constants and dielectric constants, described above. Hildebrand solubility parameters, however, do not provide information about which solvent characteristics are responsible for interrupting cohesive CNC-CNC interactions. ${ }^{68}$ For example, the cohesive energy density does not suggest why acetonitrile, a nonhydrogen bonding solvent, equally swells CNC films as ethanol, a hydrogen bonding solvent. To this end we used the Hansen solubility parameters, which separate the Hildebrand solubility parameter into its respective dispersive, polar, and hydrogen bonding components, as a measure of effective solvent properties. Although swelling observed in methanol, acetonitrile, isopropanol and ethanol are statistically within the same range, plotting

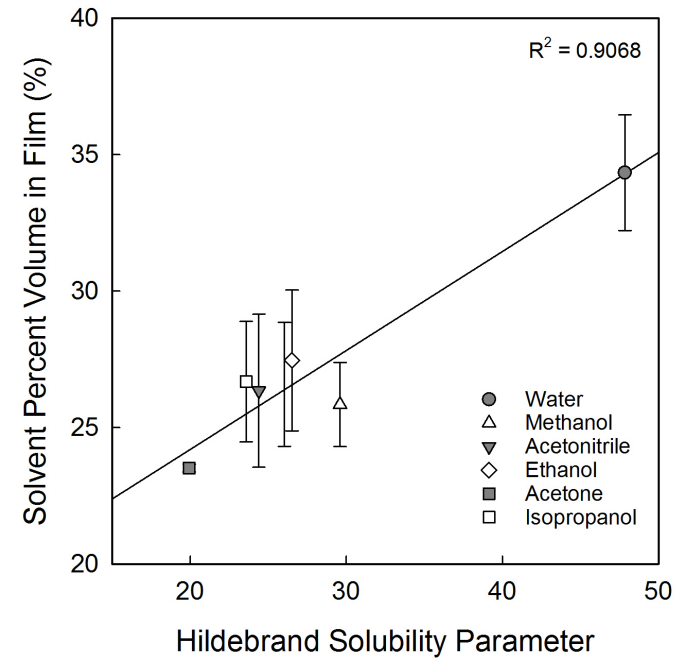

Figure 5: Percent volume of solvent in swollen CNC film versus Hildebrand solubility parameters. Line represents linear regression best fit with correlation coefficient, $\mathrm{R}^{2}=0.9068$. individual volume percentages against the Hansen solubility parameters helps' establish general trends in solvent parameters (see ESI Table S2 for theoretical Hildebrand and Hansen solubility parameters). Figure 6 presents the relationship between the solvent volume fraction in swollen CNC films and the components

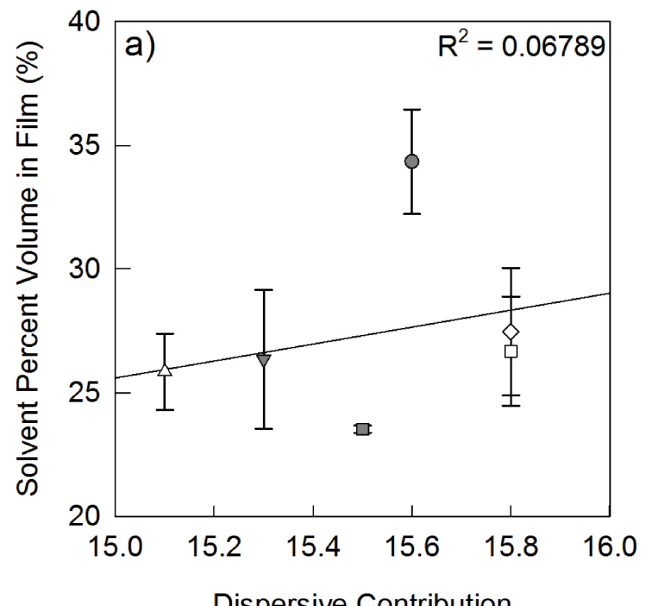

Dispersive Contribution
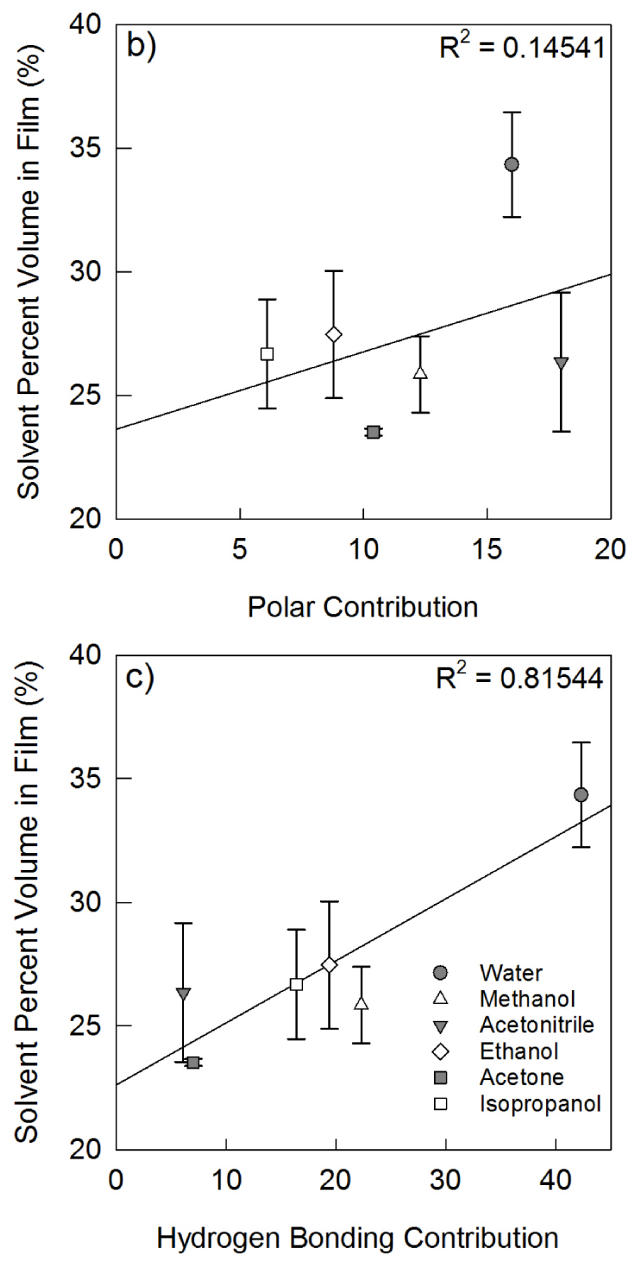

Figure 6: Percent volume of solvent in swollen CNC films for (a) dispersive, (b) polar and (c) hydrogen bonding Hansen solubility parameters. Lines represent linear regression best fits with correlation coefficient, $R^{2}$. The legend in (c) applies to all figures, (a)-(c). 
of the Hansen solubility parameters. Film swelling has a weak correlation to the dispersive and polar components (Figure 6a and 6b) however swelling does increase with an increase in the hydrogen bonding component $\left(R^{2}=0.8154\right.$, Figure $\left.6 c\right)$. This is attributed to the abundance of hydrogen bonding sites at the CNC surface and that in liquid CNC-CNC hydrogen bonds are understood to be replaced with CNC-solvent hydrogen bonds. Despite this trend, this does not demonstrate why acetonitrile is as effective as ethanol. Although there is a lack of agreement overall with polar component of the Hansen solubility parameters, the large polarity (and dielectric constant) of acetonitrile indicates that it can interrupt cohesive particle-particle interaction without hydrogen bonding. Additionally, we observe that acetone with poor hydrogen bonding capabilities and low polarity does not effectively increase the volume of the films contrasting water with high polarity and hydrogen bonding. Extending this to nanocomposites, we suggest that agglomerates will persist in non-polar polymer matrices incapable of hydrogen bonding, such as polyethylene, even under high shear possibly because of the inability for the polymer to effectively interrupt cohesive particle-particle interactions. ${ }^{22}$ Melt processed $\mathrm{CNC} /$ polyvinyl alcohol composites have been reported with improved CNC dispersibility and mechanical performance suggesting that the polymer's ability to form hydrogen bonds is critical. $^{69}$

\section{Film Stability and Particle-Particle Spacing}

Strong, attractive van der Waals forces between high aspect ratio nanoparticles at nanometer separations are well documented to be responsible for aggregates and agglomerates in nanocomposites, ${ }^{70,71}$ and we believe we have explicitly demonstrated that this is also the case for CNCs, as discussed below. Because CNC films remain stable throughout the swelling measurements even after CNC-CNC hydrogen bonds have been replaced with CNCsolvent hydrogen bonds we recognize that other short range forces are responsible for the strong particle-particle cohesion. Within swollen CNC films, the largest volume increase observed was $14 \pm$ $2 \%$ or a $5.6 \pm 0.6 \mathrm{~nm}$ thickness increase (assuming that all swelling occurs in the vertical direction due to the O-ring clamp used in the experimental setup which restricts lateral swelling) for a $40 \mathrm{~nm}$ thick CNC film in water. Assuming CNCs lay flat in distinct layers and have a square prism geometry with an average cross section of 8 $\mathrm{nm}$, the volume increase in swollen films can be equated to a 1.2$1.6 \mathrm{~nm}$ vertical spacing between each CNC (when distributed evenly) which is in excellent agreement with CNC films swollen in humid environments. ${ }^{33}$ This spacing is significantly greater than any hydrogen bonding length and is large enough to contain 4-6 molecular layers of water.

We suggest that wetting of the CNC surface induces hydration forces, which are sufficient to overcome van der Waals forces over this narrow spacing. Forces at these separations $(<2 \mathrm{~nm})$ have been measured by Pashley and Israelachvili to extend ca. $1.7 \mathrm{~nm}$ from mica surfaces or 6-7 molecular layers in good agreement with the work presented here. ${ }^{72}$ Since we expect no physical entanglements between rigid rod-like particles, beyond a spacing of $1.6 \mathrm{~nm}$ hydration effects at the CNC surface cannot overcome attractive forces and thus indicate that van der Waals forces are likely responsible for holding the films intact. Exploring this, the crossed and parallel configuration geometries of CNCs (approximated as cylinders) were averaged and Figure 7 presents simulated van der Waals attraction, electrostatic double layer repulsion, and the total combined contributions (i.e., DLVO) interaction potential profiles

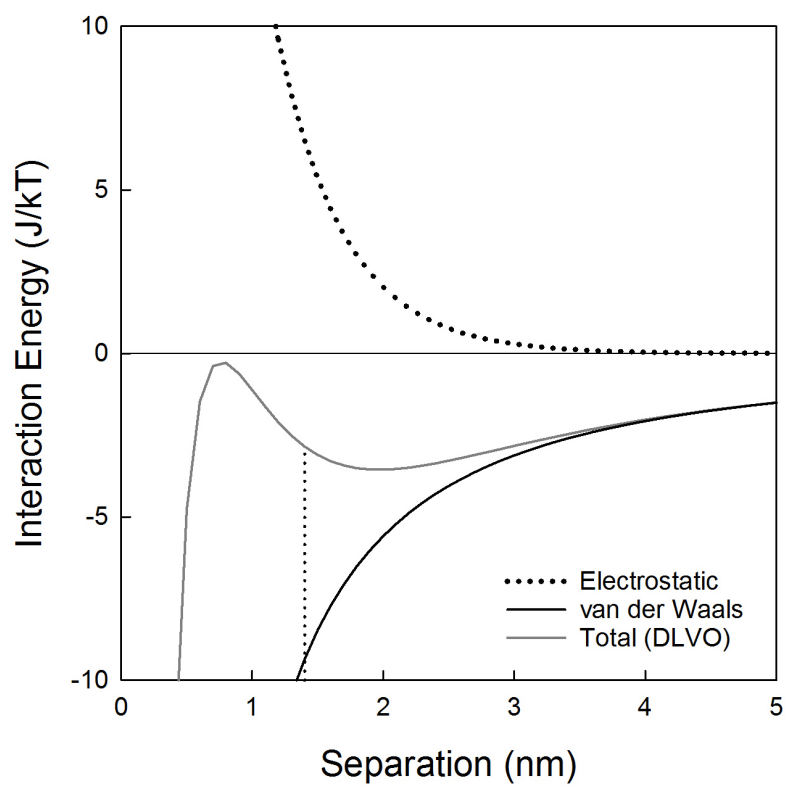

Figure 7: Simulated average attractive van der Waals energy between crossed and parallel CNCs in $0.35 \mathrm{M}$ aqueous medium, the electrostatic double layer repulsion, and the total (DLVO) interaction energy as a function of nanoparticle separation. The vertical dashed line shows the average particle separation observed from the CNC swelling experiments.

(Details in ESI.). The van der Waals contribution was calculated using a Hamaker constant of $8 \times 10^{-21} \mathrm{~J}$ as measured by Bergström et al. for cellulose in water. ${ }^{73}$ Electrostatic double layer repulsion between CNCs was calculated following Buining et al. ${ }^{74}$ assuming constant surface potential and using a surface potential for CNCs of $-18 \mathrm{mV}$ as measured by Stiernstedt et al. ${ }^{75}$ We note that the surface potential has a significant impact on the calculated interaction energy and is detailed further in the ESI. Importantly, the ionic strength due to $\mathrm{Na}^{+}$counterions within the swollen CNC film was calculated to be $0.35 \mathrm{M}$ based on the sulfate half ester content of the CNCs and water occupying $34 \%$ of the film volume (divided by 2 because only the counterions are mobile). At high ionic strengths, as is the case within the CNC film, the onset of electrostatic repulsion occurs at small separations $(<3 \mathrm{~nm})$ where attractive van der Waals forces are larger in magnitude and thus dominate. In fact, at no point does the combined DLVO interaction reach repulsive energies since the ionic strength at the initial stages of swelling is even larger than the completely swollen CNC films. (See ESI Figure S7 for visualization of the effect of ionic strength in this range on DLVO interaction energy.)

The largest CNC particle-particle spacing we observe experimentally due to swelling is $1.6 \mathrm{~nm}$ which is well within the attractive DLVO regime according to Figure 7 . At the average CNC spacing of $1.4 \mathrm{~nm}$ (dotted line, Figure 7), DLVO forces are greater than the average kinetic energy of $3 k T / 2$ (where $k$ is the Boltzmann constant and T is temperature in Kelvin). This implies that CNCs do not have sufficient thermal energy to overcome the attractive forces holding them together which demonstrates why external energy, such as sonication, is needed to fully disperse freeze dried or spray dried CNCs in water. ${ }^{21}$ As the electrostatic double layer forces play a minor role at these particle-particle separations and ionic strengths, we attribute CNC particle cohesion in the wet state primarily to van der Waals forces. 
We can furthermore extend the trend in Figure 5 to predict a Hildebrand "dispersibility" parameter for CNCs based on DLVO theory: if CNCs must be separated by more than $5 \mathrm{~nm}$ to overcome the attractive regime $(>-3 k \mathrm{~T} / 2)$ this implies a swelling of over $50 \%$ solvent volume in the film. This would correspond to a Hildebrand parameter of $150 \mathrm{MPa}^{1 / 2}$ for CNCs which is significantly greater than water $\left(47.8 \mathrm{MPa}^{1 / 2}\right)$, and much larger than common hydrophobic polymers (ESI Table S2). While this result exemplifies the difficulties associated with dispersing particles in nanocomposites these SPR swelling measurements can be easily extended to predict the dispersibility of surface modified CNCs as well as other types of nanoparticles.

\section{Conclusion}

In summary, this work demonstrates that SPR as a technique is capable of probing particle-particle cohesion at the nanometer scale in a variety of solvents. Specifically CNC-CNC particle interactions were explored via film swelling in aqueous and nonaqueous solvents. The hydrogen bonding ability of the solvent was observed to be the most correlated factor (albeit a weak correlation) in film swelling however, highly polar acetonitrile was equally effective as alcohols at screening interparticle interactions. Film swelling is proposed to occur as the solvent fills the porous structure of the film followed by enthalpic wetting of individual CNCs. This wetting replaces CNC-CNC hydrogen bonds with CNCsolvent hydrogen bonds and interrupts cohesive particle-particle interactions. In all solvents, the van der Waals forces between CNC particles maintained the particle-particle cohesion and the overall stability of the CNC films. The largest spacing observed suggests 4-6 molecular layers of water are present between each CNC, in excellent agreement with QCM-D measurements of CNCs in humid environments. ${ }^{33}$ From this work we predict that to produce welldispersed CNC nanocomposites (with unmodified CNCs), polymers likely require hydrogen bonding or polar components that can interrupt CNC-CNC bonding and the input of external energy must be sufficient to overcome van der Waals forces.

\section{Acknowledgements}

Funding from the Natural Sciences and Engineering Research Council of Canada, Industrial Postgraduate Scholarship program sponsored by Cabot Corporation is gratefully acknowledged. The authors also thank S. Kedzior, H. Marway, K. De France, D. LeClair and L. Poole for useful discussions. Professors R. Pelton, A. Guarne and J. Moran-Mirabal are thanked for sharing equipment to characterize CNCs and prepare films. The Biointerfaces Institute and the Brockhouse Institute for Materials Research at McMaster University are acknowledged for support and equipment.

\section{References}

1 J. Jancar, J. F. Douglas, F. W. Starr, S. K. Kumar, P. Cassagnau, A. J. Lesser, S. S. Sternstein and M. J. Buehler, Polymer (Guildf)., 2010, 51, 3321-3343.

2 P. Akcora, H. Liu, S. K. Kumar, J. Moll, Y. Li, B. C. Benicewicz, L. S. Schadler, D. Acehan, A. Z. Panagiotopoulos, V. Pryamitsyn, V. Ganesan, J. Ilavsky, P. Thiyagarajan, R. H. Colby and J. F. Douglas, Nat. Mater., 2009, 8, 354-9.
V. Khoshkava and M. R. Kamal, Biomacromolecules, 2013, 14, 3155-63.

J. O. Zoppe, M. Peresin, Y. Habibi, R. Venditti and O. Rojas, Appl. Mater. Interfaces, 2009, 1, 1996-2004.

X. Yang, E. Bakaic, T. Hoare and E. D. Cranston, Biomacromolecules, 2013, 14, 4447-55.

J. Harris, A. Hess, S. Rowan, C. Weder, C. Zorman, D. Tyler and J. Capadona, J. Neural Eng., 2011, 8, 046010.

E. E. Ureña-Benavides, G. Ao, V. A. Davis and C. L. Kitchens, Macromolecules, 2011, 44, 8990-8998.

Q. Wu, Y. Meng, S. Wang, Y. Li, S. Fu, L. Ma and D. Harper, J. Appl. Polym. Sci., 2014, 40525.

S. Tasset, B. Cathala, H. Bizot and I. Capron, RSC Adv., 2014, 4, 893.

J. O. Zoppe, R. A. Venditti and O. J. Rojas, J. Colloid Interface Sci., 2012, 369, 202-9.

G. Sèbe, F. Ham-Pichavant and G. Pecastaings, Biomacromolecules, 2013, 14, 2937-44.

Z. Hu, S. Ballinger, R. Pelton and E. D. Cranston, J. Colloid Interface Sci., 2014, 439, 139-148.

S. J. Eichhorn, A. Dufresne, M. Aranguren, N. E. Marcovich, J. R. Capadona, S. J. Rowan, C. Weder, W. Thielemans, M. Roman, S. Renneckar, W. Gindl, S. Veigel, J. Keckes, H. Yano, K. Abe, M. Nogi, A. N. Nakagaito, A. Mangalam, J. Simonsen, A. S. Benight, A. Bismarck, L. A. Berglund and T. Peijs, J. Mater. Sci., 2009, 45, 1-33.

S. J. Eichhorn, Soft Matter, 2011, 7, 303.

M. Mariano, N. El Kissi and A. Dufresne, J. Polym. Sci. Part B Polym. Phys., 2014, 52, 791-806.

R. J. Moon, A. Martini, J. Nairn, J. Simonsen and J. Youngblood, Chem. Soc. Rev., 2011, 40, 3941-94. S. J. Eichhorn, C. A. Baillie, N. Zafeiropoulos, L. Y. Mwaikambo, M. P. Ansell, A. Dufresne, K. M. Entwistle, P. J. Herrera-Franco, G. C. Escamilla, L. Groom, M. Hughes, C. Hill, T. G. Rials and P. M. Wild, J. Mater. Sci., 2001, 36, 2107-2131.

J. F. Revol, H. Bradford, J. Giasson, R. H. Marchessault and D. G. Gray, Int. J. Biol. Macromol., 1992, 14, 170-2. Y. Habibi, L. A. Lucia and O. J. Rojas, Chem. Rev., 2010, 110, 3479-500.

X. M. Dong and D. G. Gray, Langmuir, 1997, 13, 2404-2409. S. Beck, J. Bouchard and R. Berry, Biomacromolecules, 2012, 13, 1486-1494.

A. Junior de Menezes, G. Siqueira, A. A. S. Curvelo and A. Dufresne, Polymer (Guildf)., 2009, 50, 4552-4563.

N. Lin and A. Dufresne, Macromolecules, 2013, 46, 55705583.

Y. Habibi, Chem. Soc. Rev., 2014, 43, 1519-42.

Future Markets Inc., The Global Market for Nanocellulose to 2020, 2013.

E. Kontturi, T. Tammelin and M. Österberg, Chem. Soc. Rev., 2006, 35, 1287-1304.

Z. Hu, E. D. Cranston, R. Ng and R. Pelton, Langmuir, 2014. C. V. Cerclier, A. Guyomard-Lack, F. Cousin, B. Jean, E. Bonnin, B. Cathala and C. Moreau, Biomacromolecules, 2013, 14, 3599-3609.

K. S. Kontturi, E. Kontturi and J. Laine, J. Mater. Chem. A, 2013, 1, 13655.

C. Aulin, S. Ahola, P. Josefsson, T. Nishino, Y. Hirose, M. Österberg and L. Wågberg, Langmuir, 2009, 25, 7675-85. F. Jiang, J. Kittle, X. Tan, A. Esker and M. Roman, Langmuir, 2013. 
J. D. Kittle, X. Du, F. Jiang, C. Qian, T. Heinze, M. Roman and

A. R. Esker, Biomacromolecules, 2011, 12, 2881-7.

33

Einivaara, M. Faustini, T. Tammelin and E. Kontturi, Langmuir, 2015, 31, 12170-12176.

A. N. Raegen, K. Reiter, A. Dion, A. J. Clarke, J. Lipkowski and J. R. Dutcher, Anal. Chem., 2014, 86, 3346-54. Z. Liu, H. Choi, P. Gatenholm and A. R. Esker, Langmuir, 2011, 27, 8718-28. . Wu, S. Turgman-Cohen, J. Genzer, T. W. Theyson and O. J. Rojas, Langmuir, 2010, 26, 9565-9574.

37 S. G. Allen, O. M. Tanchak, A. Quirk, A. N. Raegen, K. Reiter, R. Whitney, A. J. Clarke, J. Lipkowski and J. R. Dutcher, Anal. Methods, 2012, 4, 3238.

O. Grigoray, H. Wondraczek, E. Heikkilä, P. Fardim and T. Heinze, Carbohydr. Polym., 2014, 111, 280-287. K. Junka, J. Guo, I. Filpponen, J. Laine and O. J. Rojas, Biomacromolecules, 2014, 15, 876-881. Rojas, Biomacromolecules, 2011, 12, 4311-4318. H. Orelma, L. O. Morales, L.-S. Johansson, I. C. Hoeger, I. Filpponen, C. Castro, O. J. Rojas and J. Laine, RSC Adv., 2014, 4, 51440-51450.

H. Orelma, T. Teerinen, L. S. Johansson, S. Holappa and J. Laine, Biomacromolecules, 2012, 13, 1051-1058. S. Ahola, J. Salmi, L. S. Johansson, J. Laine and M. Österberg, Biomacromolecules, 2008, 9, 1273-1282. S. E. Gradwell, S. Renneckar, A. R. Esker, T. Heinze, P. Gatenholm, C. Vaca-Garcia and W. Glasser, Comptes Rendus - Biol., 2004, 327, 945-953. X. Dong, T. Kimura, J. Revol and D. G. Gray, Langmuir 1996, 2076-2082.

D. Viet, S. Beck-Candanedo and D. G. Gray, Cellulose, 2006, 14, 109-113. E. D. Cranston and D. G. Gray, Biomacromolecules, 2006, 7 2522-30.

48 M. Labet and W. Thielemans, Cellulose, 2011, 18, 607-617.

49 S. Beck, M. Méthot and J. Bouchard, Cellulose, 2014, 22, 101-116.

50 N. Granqvist, H. Liang, T. Laurila, J. Sadowski, M. Yliperttula and T. Viitala, Langmuir, 2013, 29, 8561-71. S. N. Kasarova, N. G. Sultanova, C. D. Ivanov and I. D. Nikolov, Opt. Mater. (Amst)., 2007, 29, 1481-1490. W. Ogieglo, H. Wormeester, K.-J. Eichhorn, M. Wessling and N. E. Benes, Prog. Polym. Sci., 2015, 42, 42-78. Polymer (Guildf)., 2014, 55, 1737-1744 B. W. Rowe, B. D. Freeman and D. R. Paul, Macromolecules, 2007, 40, 2806-2813. M. Erdoğan, I. Capan, C. Tarimci and A. K. Hassan, J. Colloid Interface Sci., 2008, 323, 235-41. . Erdogan, R. Capan and F. Davis, Sensors Actuators B Chem., 2010, 145, 66-70. Z. Ozbek, M. Erdoğan and R. Capan, Sensors Actuators B Chem., 2014, 196, 328-335.

58 T. Akimoto, S. Sasaki, K. Ikebukuro and I. Karube, Appl. Opt., 1999.

59 C. D. Edgar and D. G. Gray, Cellulose, 2003, 10, 299-306.

60 E. D. Cranston and D. G. Gray, Colloids Surfaces $A$ Physicochem. Eng. Asp., 2008, 325, 44-51.

61 M. M. Braun and L. Pilon, Thin Solid Films, 2006, 496, 505514.
S. Fält, L. Wågberg and E. L. Vesterlind, Langmuir, 2003, 19, 7895-7903.

T. Viitala, H. Liang, M. Gupta, T. Zwinger, M. Yliperttula and A. Bunker, J. Colloid Interface Sci., 2012, 378, 251-259. H. Hatakeyama and T. Hatakeyama, Thermochim. Acta, 1998, 308, 3-22.

R. J. Maurer, A. F. Sax and V. Ribitsch, Cellulose, 2013, 20, 25-42.

M. Ioelovich, Sci. Isreal Technol. Advantages, 2011, 13, 3544.

C. M. Hansen, Prog. Org. Coatings, 2004, 51, 77-84. L. a Errede, Macromolecules, 1986, 1525, 1522-1525. W. Zhang, X. He, C. Li, X. Zhang, C. Lu, X. Zhang and Y. Deng, Cellulose, 2014, 21, 485-494.

Y. Y. Huang and E. M. Terentjev, Polymers (Basel)., 2012, 4, 275-295.

V. Singh, D. Joung, L. Zhai, S. Das, S. I. Khondaker and S. Seal, Prog. Mater. Sci., 2011, 56, 1178-1271.

R. M. Pashley and J. N. Israelachvili, J. Colloid Interface Sci., 1984, 101, 511-523.

L. Bergström, S. Stemme, T. Dahlfors, H. Arwin and L. Ödberg, Cellulose, 1999, 6, 1-13.

P. Buining, A. Philipse and H. Lekkerkerker, Langmuir, 1994, 2106-2114.

J. Stiernstedt, N. Nordgren, L. Wågberg, H. Brumer, D. G. Gray and M. W. Rutland, J. Colloid Interface Sci., 2006, 303, 117-23. 